\title{
IDENTIFICATION AND VERIFICATION OF VEHICLE USING RFID TECHNIQUE
}

\author{
$\overline{\overline{\text { AQIB MEHMOOD }^{1}, \text { MUMTAZ ALI }^{2} \text { RAMEEZ AHMAD }}{ }^{1,} \text { SAYED IRFAN }^{1}, \text { HABIB UR RAHMAN }^{1}}$ \\ ${ }^{1}$ Department of Computing and Technology Abasyn University Peshawar, Pakistan \\ ${ }^{2}$ Department of Computer Science, City University Peshawar, Pakistan \\ E-mail: Aqibmehmood7@gmail.com
}

\begin{abstract}
The number of vehicles/traffic is increasing exponentially day-by-day, due to which automatic vehicles identification/monitoring system is being paid significance all over the world. Different countries in the world are using various types of automatic systems for traffic control, vehicles identification. Number plate recognition (NPR) is an authentic-time embedded system which frequently recognition the number plate of vehicle. Pervious systems are using only for identification of vehicle. The proposed system cumulates both the RFID (Radio Frequency Identification) and NPR systems for the identification and verification of vehicle.
\end{abstract}

Keyword: NPR, E-tag, RFID

1. Introduction: Different countries makes use of specific process for identification of vehicle \& for traffic control, targeted visitors investigation, checking against the law activities, security control connected with restricted areas, traffic law, enforcements agencies, toll collection \& car parks management \& thus Pakistan also has a process of assigning different numbers to vehicles. These matchless numbers plates are allocated on the vehicles by RTO (Regional Transport Office). It is believed that they now have currently over 50 percent a billion of cars on the roads worldwide. All individuals vehicles have identification number because their primary identifier. The vehicle number is truly an identification number, which positions the legal number to engage in the public traffic. All vehicle must have its Number written with a Number plate placed onto its body (at least on the back side) and not any vehicle without properly placed, will observable \& effectively understandable number plate have to be powered by the roads. To process, sort or analyses knowledge everyone considers using computers. If the knowledge has already been in the computer lots of these tasks are simple to be carried out. It is naturally that the Number is an important identification knowledge a computer technique must to treat when managing vehicles. NPR provides automated access from the content of the number plate for computers managing databases \& processing knowledge of vehicle movements.

NPR technology is continuously ahead popularity, particularly in safety and traffic controller system. The systems are applied often for access control in parking area, law enforcement, stolen vehicle detection, traffic controlling and toll collection. Although NPR and RFID system is being used separately in various countries from last four decades for aforementioned purposes, but there is no such system to verify the gathered information. NPR is an authentic-time implanted system which automatically recognizes the number plate of vehicle. Such systems need the localization of number plate area in order to recognize the fonts present on it.

Number Plate Recognition System (NPRS) is exciting region in the area of image processing and computer vision. Various systems such as law enforcement, parking and vehicle ticketing systems, automated toll collection, automated vehicle access in secure system.

A great deal of work has been done in this field; however none of them proposed verification. This served as a motivation to develop an identification and verification system as a dedicated hardware unit for the verification and identification of the vehicle Number plates [6].

2. Literature Review: The most important and also the most worrying portion of any number plate recognition system [11] is that the recognition and extraction of the vehicle number that directly affected overall systems 
accuracy. Presence of noise, distorting within the image, irregular illumination, blurred and foggy environments build the job even tougher. During this work we tend to suggest a close and different technique for correctly police investigate the vehicle number plates. The projected system will work very correctly in effectively any atmosphere, daytime, and circumstances.

There are certain foreign, national or native standards for vehicles. In China, the fundamental standards [12] for the volume plate are assumed. Certain native co-operations like European Public (EU), have number plates that outline the country, the place of registration, etc. during this text, Chinese, Pakistani, and Kuwaiti number plates are coated. The problem of automatic NPR is being discuses later in 90's [5], [8], [10].

The capture image being first processed to enhance and improve boundary line-information by mistreatment such algorithms because the gradient filter, and leading to a picture shaped of edges. The image so processed reborn to its binary matching half so processed by sure algorithms, like Hough rework, to observe lines. Eventually, couples of 2-parallel lines were thought of as a plate elect [6], [11]. Alternative technique supported the morphology of substances in a picture [1], [7].

This technique attentions on certain relevant properties of car plate pictures like their illumination, contrast, regularity, positions, etc. owing to these options, this technique might be accustomed notice the parallel properties in a very sure image and find the location of variety plate areas. The third technique was supported applied math properties of text [3], [4]. During this method, text regions were exposed mistreatment applied math properties of text just like variance of grey level, variety of boundaries, edge thicknesses within the area, etc. This approach was unremarkably utilized in finding text in pictures, and will rather be used for locating and designating candidate variety plate areas as they embrace letters and numbers.

There is variety of different approaches regarding this downside specializing in police investigation NPR mistreatment computing and genetic algorithms [2], [9]. These systems used edge observation and edge information so artificial intelligence techniques to detect the position of the amount plate elect space. All systems mentioned higher than have some quite limits as an example they're plate size dependent, color reliant on, work solely in sure conditions or atmosphere similar indoor pictures etc. the tactic that we tend to suggesting the freelance of color, size, location and position of the amount number plate of the vehicle.

A lot of studies and different approaches have been suggested. The major work is done on plate localization, which are precise to the NPR area. But they have no study about verification and extraction in same system. The model implementation expected a clear number plate text and more tests need to be shown on this system. The text extraction process involves localization, improvement and recognition of text blocks in a given image. Number plate Recognition (NPR) systems can be recognized as an application of Text Information Extraction (TIE) algorithms. As the application is specific to Number plate images, NPR text extraction process can leverage particular visual features and constraints. Different methods have been proposed on NPR text extraction and each uses a specific set of perceptible features to emphasize the text areas.

3. Problem definition: Number extraction from number plates involves some image processing and computer vision processes to be applied within a solo system. Text segmentation and recognition operations are used to determine the number plate in a specified image. Few of the earlier studies contain all the steps of a typical NPR system, from image acquisition to identification.

Similarly the RFID system used in different types of fields. RFID system used in various countries for different types of areas like car parking, toll collection, employee attendance. RFID is additionally utilized for examining and controlling security persons themselves. Application delivers barriers for patrolling the security protector. Checkpoints are basically a RFID tag which security protector needs to scan during their sequential watch through the reader. Although, both the systems are working very fine in their respective domains but if they are analyzed for their work, both of them are working for identification and there is no verification found. So the main problem that was identified is the verification of the identified Number Plate or RFID client.

4. Problem solution: Number plate Recognition (NPR) and verification system is the combination of image processing, and Radio Frequency Identification (RFID) used to recognize and authenticate vehicles by their number plates. The extracted text is used for identification and RFID information for verification. This technology requires installing RFID tag on the vehicle only.

Technique of RFID-tag system which will extract the cars specification, so by this we can compare the information of RFID-tag by the RFID reader. Main goal of this work is to provide a method efficient in recognizing number plates in any conditions. Number plate Recognition (NPR) is a combination of image processing, character segmentation and recognition skills used to recognize vehicles by their number plates. Since the number plate information is used for recognition, technology needs no extra hardware to be fixed on vehicles. NPR technology is continually ahead popularity, especially in safety and traffic controlling systems. NPR systems 
are utilized regularly for access control in parking areas, law enforcement, stolen car detection, traffic control, automatic toll collection and marketing work.

Suggested technique for authentication of number plates by RFID-tag and RFID system which is already implemented in different areas. RFID tags annexed to vehicles vehicle 'ID' mapped vehicle owner, group of owners, sanctioned personnel Scanning mechanism at entry/exit points for vehicle identification (RFID scanners to detect tags on vehicles) person identification, if required (RFID cards issued to vehicle owners).

4.1. Number Plate Recognition Systems. Recent improvements in technology placed best cameras, and application of high reflective backgrounds in number plate have better accuracy of NPR systems. After the recognition method, the data can be more tested within remote databases and stored for future referencing. Usual NPR system is collection of some hardware and software components as showed.

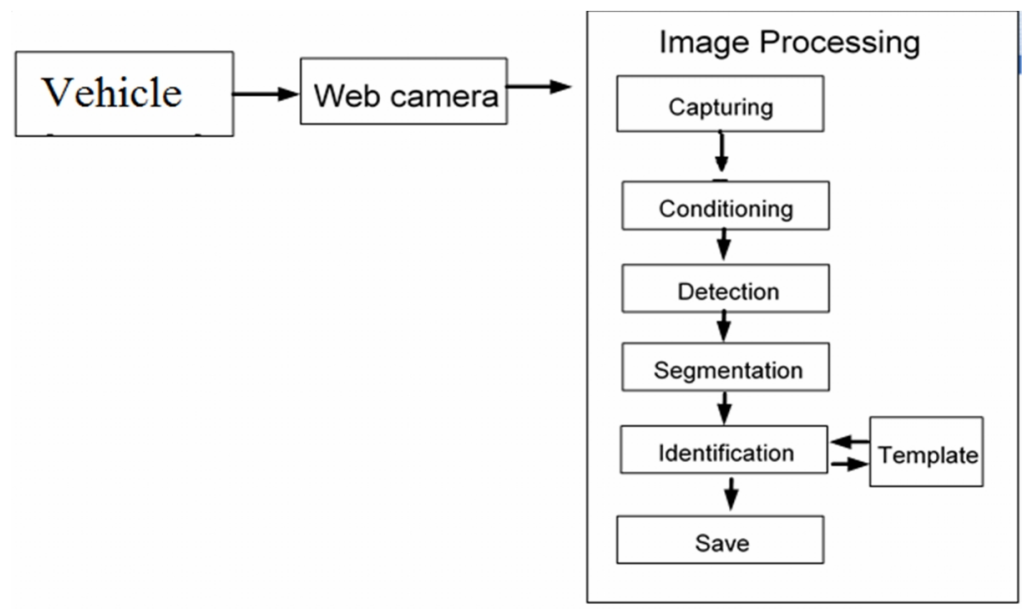

Figure 1: Block diagram of (NPR)

Usual NPR system works, when the vehicle came at the protected area, it starts the cycle by walking over a magnetic ring, sensor where RFID reader placed (which is the vehicle sensor). The detector senses the vehicle and refers signals to the NPR unit. The NPR component activates light and capture image of the front rear plates of vehicle through the camera. The system examines the image with different algorithms, improves the image, identifies the plate location, extracts the plate string, and recognizes the fonts. The extracted number plate information will registered, stored along with the taken image are used for verification depends on the NPR application. The recognition is not unqualified and may contain errors due to problems in some of the NPR phases. Applications require working proper verification and control approaches in order to pay for the possible problems. If the system not detected tag than number plates are not properly recognized are processed.

4.2. Segmentation Segmentation is useful in an array of computer vision applications, but fully automatic segmentation remains an arduous issue. There has been extensive work with picture segmentation and the broader issue of files partitioning. The aim of segmentation is usually to find groups which are usually both homogeneous, such that information within the same group are Similar \& well separated, so that information in different teams are dissimilar. Lots of approaches are actually proposed, which may become broadly categorized as often contourbased, region-based or combining both. On the whole, the taken picture may cover several number menu candidates. The recognition algorithm usually clips some bands, as well as some plates from every band. There are predefined costs of number of applicants, which are discovered by study of predictions. The value is compatible to nine. There are usually some heuristics, which are used to choose the rate of certain applicants in line with their properties. These heuristics are actually selected commercial hoc in the coursework of the useful testing. The recognition sense categories candidates in line with their rate from the best choice to the smallest appropriate. The best appropriate candidate is scanned by way of a deeper heuristic analysis. The particular proper analysis certainly allows, or discards the prospect. It examines single characters; this type of study consumes large level of processor time. The simple reasoning behind analysis can be showed from the following steps:

1) Determine doable variety plate candidates or contenders.

2) Classes consistent with their price (firm by a simple heuristics).

3) Changed the primary plate from the list with the most effective price.

4) Phase and analyze it by a deeper examine (time consuming). 
5) If the proper study rejects the plate, come back to the step three.

Priority choice and basic heuristic analysis of bands the basic analysis is employed to test the value of candidates, and to kind them consistent with this price. There are many freelance heuristics as shown in the table 1, which might be wont to evaluate the value $\alpha$ me. The heuristics is used singly, or they will be joint along to calculate Associate in nursing overall price of candidate by a weighted sum:

$$
\alpha=0.15 \cdot \alpha_{1}+0.25 \cdot \alpha_{2}+0.4 \cdot \alpha_{3}+0.4 \cdot \alpha_{4}
$$

Table 1. Heuristics, their design and explanation

\begin{tabular}{|c|c|c|}
\hline Heuristics & Design & Explanation \\
\hline $\begin{array}{l}\alpha_{1}=\mid y_{b 0} \\
-y_{b l} \mid\end{array}$ & $1855^{\circ}$ & $\begin{array}{l}\text { The elevation of band in pixels. Bands } \\
\text { With a minor elevation will be chosen. }\end{array}$ \\
\hline$\alpha=\frac{1}{p_{\text {y }\left(y_{\text {○а }}\right)}}$ & & $\begin{array}{l}\text { The " } p \text { y }(y \quad b \quad \mathrm{~m}) \text { " is an extreme value of top of vertical projection of } \\
\text { picture, which parallels to the processed Band. Bands with a higher } \\
\text { amount of Vertical edges will be chosen. }\end{array}$ \\
\hline 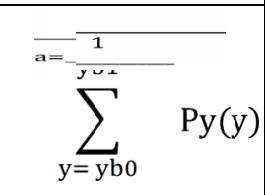 & 然 & $\begin{array}{l}\text { This heuristics is alike to the earlier one, but it reflects not only the value } \\
\text { of the maximum peak, but a value of area below the graph among point's y } \\
\text { b0 and y b1. These points describe a vertical Location of the estimated } \\
\text { band. }\end{array}$ \\
\hline
\end{tabular}

4.3. Methodology. The proposed technique for the recognition and verification of number plates consists of the following processes, as shown in Fig. 1.

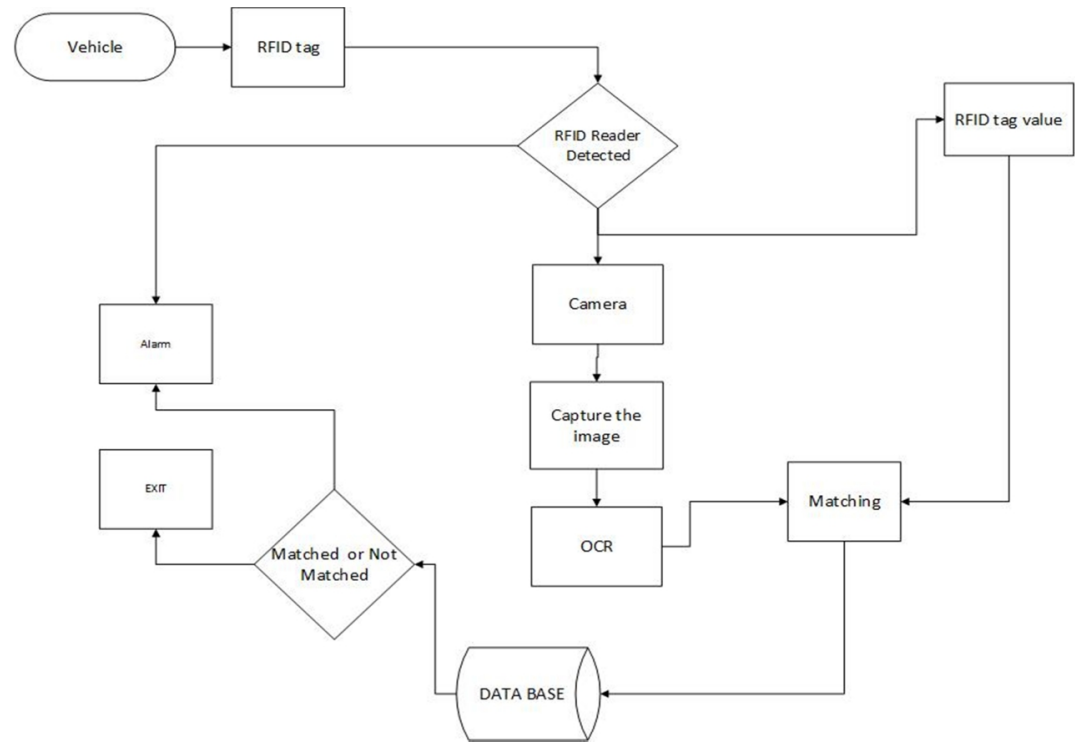

Fig 1: Data flow diagram

Steps for identification and verification

a. Input Image from file

- Capture image from Camera

b. Change image into binary 
- Classify the intensity of the image

- Change image into gray scale

- Compute suitable threshold value for the image

- Change the image into binary image by the calculated threshold

c. Identifying Number plate region

- Fill small holes with amounts of number plate so that its area will be large to separate from figure.

- Control width and height of the image.

- Use the identifying number plate region for both horizontal and vertical direction.

- Crop the necessary region.

d. Segmentation

- Mesh the noise level existing in the image.

- Slide the plate region in that way only numbers of plate region extracted.

- Isolated every character on the number plate.

e. Number Recognition

- Generate the pattern file from the stored pattern images.

- Resize image grown from segmentation to the size of pattern.

- Match every character with the patterns.

- Store the finest matched character.

f. Save to file in certain format

- Create a file in write mode.

- Store the character grown from the number recognition method to text file in certain format.

g. Match Module

- RFID reader collected the data from RFID-tag.

- The data in RFID-tag will extracted.

- RFID value and data in text file will matched with each other.

- Either values will matched or not match it will show status.

4.4. Result

- Application Close.

\section{a. Verification module}

In this module the both values (RFID tag value and extracted values which taken from image) will checked, If the RFID value and (OCR) extracted value same the status show matched and otherwise the will not matched status appear as not matched. Result will show in following Figures 4,5 and 6.

\subsection{Graphical User Interface (GUI)}

When RFID tag detected camera automatically capture the image form car and display in another axes, after that process will be started. When processing will completed the extracted value which taken from image will exposed in figure after that extracted value will save in data base. The result exposed in following Figures 2 and 3.

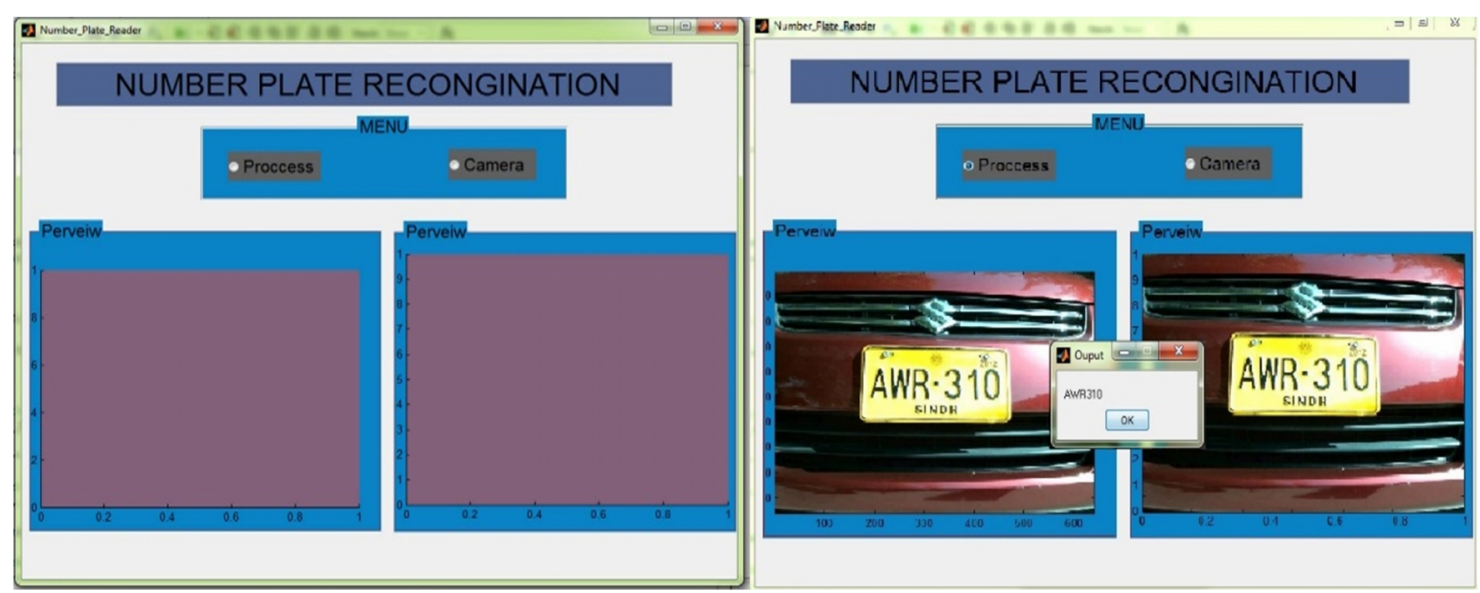

Figure 2: GUI guide

Figure 3: (OCR) extracted output 


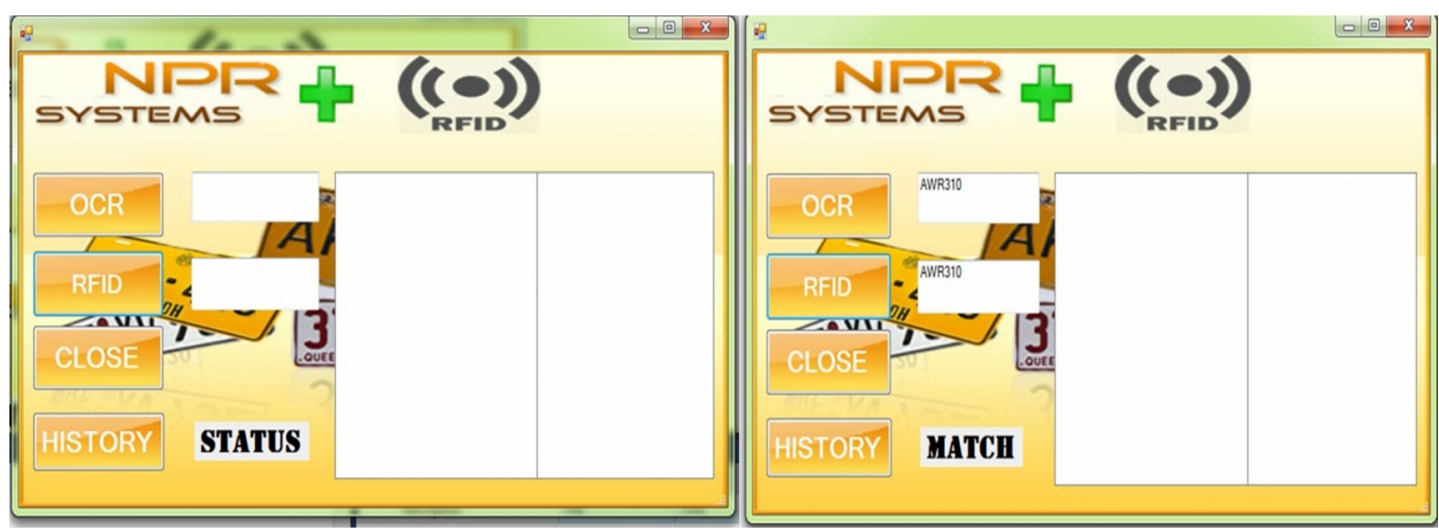

Figure 4: RIFD and OCR matching

Figure 5: show matched view

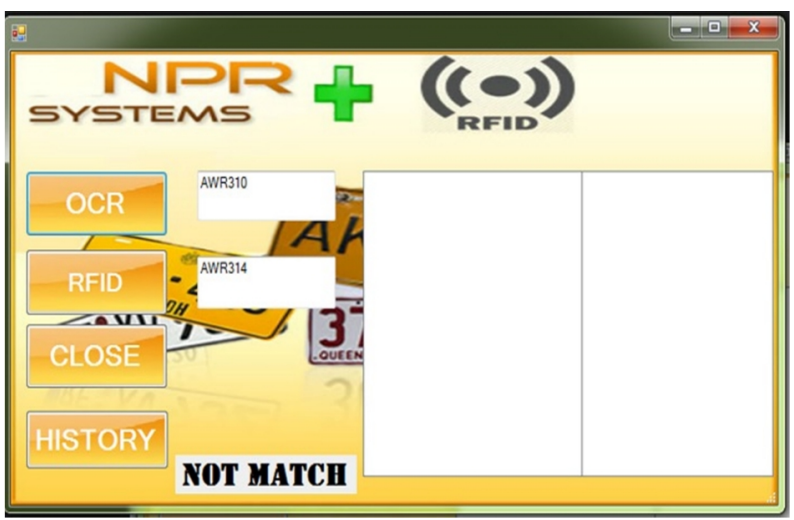

Figure 6: If RIFD and OCR not matching

Table 2. Characteristic of Misreads

\begin{tabular}{|c|c|c|}
\hline & $\begin{array}{l}\text { Percentage } \\
\text { Misreads }\end{array}$ & $\begin{array}{l}\text { Cumulative } \\
\text { percent }\end{array}$ \\
\hline Illegal font & 72.6 & 72.6 \\
\hline Marks & 23.0 & 95.6 \\
\hline Obscured & 1.8 & 97.4 \\
\hline Broken & 1.8 & 99.1 \\
\hline Screw cap & 0.9 & 100.0 \\
\hline
\end{tabular}




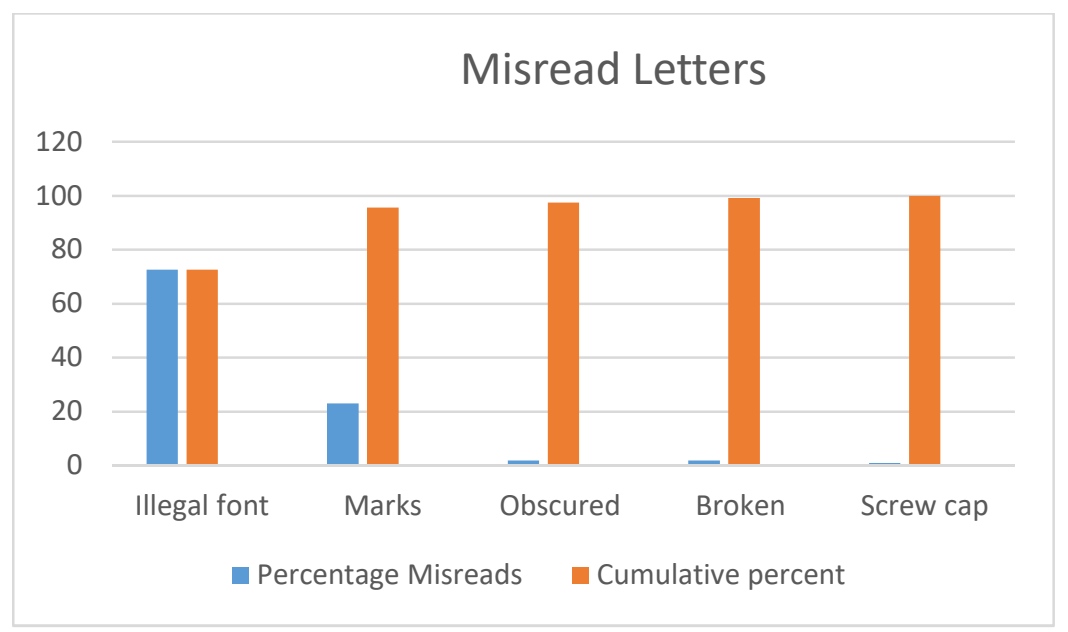

Figure 7: Position of misread letters

5. Conclusion. The work was aimed at proposing efficient, fast, simple and hardware friendly vehicle Number plate recognition system. The two modules of the project namely (i) number plate recognition system (NPRS) (ii) Radio Frequency Identification (RFID) have been individually gathered. Both the modules working parallel. Due to its expansive application area, both academic and commercial world's number plate recognition systems have received more attention. Induced in an authentic time NPRS implementation techniques and algorithms for exploring possible. Relatives are different ways to achieve results even if, at the time of execution of the selection algorithm plays a supreme role. On the consequence, some image enhancement, optimization and segmentation approaches were used through the experiments. NPR vehicle identification system very fast and accurate results can create. In this study, the shape and limit the number plate was used for optimization purposes. The rate of vehicle that they contain different fonts and character sizes are lower on the foreign number plates was quantified. In the future, additional constraints can be made faster and more accurate identification of the application. NPR character recognition system still remains the main problem. Illumination conditions and high variance due to poor image quality, this is an extremely challenging task in the domain NPR remains the subject.

\section{REFERENCE}

[1]. Hongliang, B., \& Changping, L. (2004, August). A hybrid license plate extraction method based on edge statistics and morphology. In Pattern Recognition, 2004. ICPR 2004. Proceedings of the 17th International Conference on (Vol. 2, pp. 831-834). IEEE.

[2]. Clark, P., \& Mirmehdi, M. (2000, September). Finding text regions using localised measures. In Proceedings of the 11th British Machine Vision Conference (pp. 675-684).

[3]. Clark, P., \& Mirmehdi, M. (2000). Combining statistical measures to find image text regions. In Pattern Recognition, 2000. Proceedings. 15th International Conference on (Vol. 1, pp. 450-453). IEEE.

[4]. Duan, T. D., Du, T. H., Phuoc, T. V., \& Hoang, N. V. (2005, February). Building an automatic vehicle license plate recognition system. In Proc. Int. Conf. Comput. Sci. RIVF (pp. 59-63).

[5]. Duan, T. D., Duc, D. A., \& Du, T. L. H. (2004, October). Combining Hough transform and contour algorithm for detecting vehicles' license-plates. In Intelligent Multimedia, Video and Speech Processing, 2004. Proceedings of 2004 International Symposium on (pp. 747-750). IEEE.

[6]. Castleman, K. (1993). Digital image processing

[7]. Gonzalez, R. C., \& Richard, E. (2002). Woods, digital image processing. ed: Prentice Hall Press, ISBN 0201-18075-8.

[8]. Lee, J. C. (1999). Automatic character recognition for moving and stationary vehicles and containers in reallife images. In Neural Networks, 1999. IJCNN'99. International Joint Conference on (Vol. 4, pp. 28242828). IEEE.

[9]. Parisi, R., Di Claudio, E. D., Lucarelli, G., \& Orlandi, G. (1998, June). Car plate recognition by neural networks and image processing. In Circuits and Systems, 1998. ISCAS'98. Proceedings of the 1998 IEEE International Symposium on (Vol. 3, pp. 195-198). IEEE. 
[10]. Federl, J. P. P. (1997). An Approach To Licence Plate Recognition

[11]. Remus, B. R. A. D. (2001). License plate recognition system. In Proceedings of the 3rd international conference in information, communications and signal processing (pp. 203-206).

[12]. Sulehria, H. K., Ye Zhang, D. I., \& Sulehria, A. K. (2008). Vehicle number plate recognition using mathematical morphology and neural networks. WSEAS Transactions on Computers, 7 